\title{
La dimensión socioespacial del capital social. Análisis del capital social vecinal y la eficacia colectiva en ocho conjuntos de vivienda social en Chile
}

Sebastián Ibarra. Universidad de Aysén, Coyhaique, Chile.

RESUMEN | Siguiendo el enfoque de la "espacialización” del capital social, este artículo analiza la vecindad territorial como ámbito socioespacial en el que se generan y reproducen el capital social vecinal y la eficacia colectiva. A partir del análisis de los datos de una encuesta aplicada en ocho conjuntos de vivienda social ubicados en tres regiones de Chile, esta investigación muestra la existencia de bajos niveles de confianza vecinal, el predominio de relaciones de apoyo circunstancial, y la escasez de prácticas asociativas y de cooperación. Las variables que tienen un efecto significativo sobre el capital social vecinal y la eficacia colectiva son las condiciones de acceso a la vivienda (asignatario original y propietario) y la percepción de violencia y delincuencia en el barrio. En contrapartida, la segregación socioeconómica no tiene un efecto directo sobre el capital social a nivel de los barrios analizados.

PALABRAS CLAVE | vivienda, integración social, segregación.

ABSTRACT | Drawing on the "spacialization" of social capital approach, this paper analyzes the neighborhood as the socio-spatial environment in which neighborhood social capital and collective efficacy are generated and reproduced. Based on the analysis of a survey applied in eight social housing projects located in three regions of Chile, this research shows the existence of low levels of trust among neighbors, the predominance of circumstantial support relationships and the lack of associative and cooperative practices. The variables that have a significant effect on neighborhood social capital and collective efficacy are conditions of access to housing (original assignment and ownership of housing) and perception of violence and crime in the neighborhood. In contrast, socio-economic segregation does not have a direct effect on social capital in the analyzed neighborhoods.

KEYWORDs | housing, social integration, segregation. 


\section{Introducción}

La relación entre barrios, comunidad y cohesión social ha sido objeto de análisis en las ciencias sociales desde la primera mitad del siglo xx, y ello principalmente a partir de los procesos de urbanización, los cuales configuraron un nuevo orden social basado en el individualismo y el anonimato (Forrest \& Kearns, 2001). Ya hacia finales del siglo xx y a partir de las consecuencias de los procesos de globalización, el debate en torno a tales materias adquirió un nuevo impulso. Las transformaciones en los ámbitos de la comunicación y el transporte, que tienden a romper las barreras físicas y espaciales de las relaciones sociales, han llevado a replantear el análisis de la vecindad territorial como ámbito espacial relevante en la configuración de los vínculos sociales. Por otra parte, desde la década de 1980 esta discusión se ha visto influenciada por la emergencia del concepto de capital social, el que aborda las relaciones de confianza y reciprocidad como recursos potenciales de que disponen los individuos y las comunidades (Atria, 2003).

En este contexto se ha desarrollado un amplio campo de investigación, en el que se exploran las diversas formas de articulación entre el análisis espacial y el de redes sociales (Adams, Faust, \& Lovasi, 2012). Un ámbito específico de indagación ha sido el de la "espacialización" del capital social, aproximación que se ha focalizado en analizar cómo las particularidades históricas, culturales y políticas de los espacios urbanos inciden en la (re)producción y funcionamiento de las redes de capital social. El punto de partida de estas investigaciones es el supuesto de que el capital social no se puede entender si no se considera la localización espacial en que los agentes desarrollan los vínculos sociales que lo conforman (Blokland \& Savage, 2008; Häkli \& Minca, 2009).

Tomando como referencia el campo de investigación señalado, este artículo se centra en las características de las redes de capital social en conjuntos habitacionales de vivienda social en que se acumulan altos niveles de hogares en situación de pobreza. En el caso de Chile, la cuestión de los vínculos sociales y la cohesión social a nivel de los barrios pobres ha adquirido relevancia a partir de las evaluaciones críticas de las políticas de vivienda social implementadas desde la década de 1980. Si bien estas evaluaciones han constatado el éxito cuantitativo de dichas políticas, expresado en una reducción significativa del déficit habitacional acumulado, también han planteado que ese stock de viviendas sociales construidas ha originado nuevas problemáticas sociourbanas, tales como la fragmentación urbana, la segregación residencial y los procesos de guetización (Ducci, 2005; Hidalgo, 2004; Lunecke, 2012; Sugranyes, 2005). En este contexto, se ha instalado la pregunta por el tipo de vínculos sociales que se construyen al interior de los conjuntos de vivienda social.

El análisis del capital social propuesto en este artículo considera distintos tipos de redes de relaciones que se desarrollan en los conjuntos de vivienda social, planteando al respecto una distinción conceptual entre el capital social vecinal y la eficacia colectiva. Por otra parte, en términos analíticos, este trabajo tiene dos objetivos. Como punto de partida, medir los niveles de capital social vecinal y de eficacia colectiva en los conjuntos de vivienda social estudiados, para luego, en una segunda 
instancia, analizar el efecto que tienen las variables socioespaciales de los conjuntos habitacionales (segregación residencial socioeconómica y percepción de violencia y delincuencia) e individuales (sociodemográficas, acceso a la vivienda y percepción subjetiva de aislamiento), sobre el capital social vecinal y la eficacia colectiva. Para realizar estos análisis se utilizan los datos de una encuesta aplicada a 1797 hogares pertenecientes a ocho conjuntos habitacionales de vivienda social, localizados en las regiones Metropolitana, del Maule y del Biobío.

\section{Marco conceptual}

\section{La dimensión espacial de las redes sociales:}

\section{los vínculos entre la vecindad territorial y el capital social}

En el campo de las ciencias sociales, no es una novedad que los procesos sociales se analicen tomando en consideración el contexto espacial y geográfico en el que se desarrollan (Abbott, 1997). Sin embargo, durante la última década se ha intensificado el interés por articular los enfoques del análisis espacial y de las redes sociales, ámbito dentro del cual se incluye el capital social. ${ }^{1} \mathrm{Al}$ respecto, una de las líneas de investigación más desarrolladas es la que se centra en los determinantes espaciales de las redes sociales, aproximación según la cual las personas que se encuentran reunidas en un espacio físico tienen altas probabilidades de generar vínculos entre sí (Adams, Faust, \& Lovasi, 2012). En esta línea, también se considera que la incidencia de la localización espacial sobre la conformación de los vínculos sociales no es uniforme, sino que opera de manera diferenciada según sean la escala en la que se analice y el modo en que se conceptualicen las redes sociales.

En este artículo, la configuración de las redes sociales se examina a escala barrial, específicamente en conjuntos habitacionales de vivienda social. Ello ha implicado revisar, por una parte, el rol de los barrios en la experiencia cotidiana de los individuos y su incidencia sobre diferentes procesos sociales; y por otra, la literatura sobre la relación entre los barrios de vivienda social y la conformación de las redes sociales de sus habitantes.

En relación con la discusión sobre el rol de los barrios, se pueden distinguir al menos tres enfoques de análisis. El primero sostiene la pérdida de relevancia del barrio como fuente de vínculos sociales, lo cual se explicaría por el predominio de estilos de vida individualistas y de acciones sociales más fluidas espacialmente y menos concentradas en el barrio. Desde esta perspectiva, se sostiene que en el barrio tiende a predominar un tipo de vínculos sociales que solo se activan frente a circunstancias o finalidades específicas (Forrest \& Kearns, 2001). El segundo enfoque plantea la hipótesis de que los barrios no han perdido relevancia, sino que lo que ha ocurrido es una reconfiguración de los modos en que los individuos construyen una identidad y un sentido de pertenencia con los barrios que habitan. Por lo tanto, el rol de los barrios es el de articulación entre el espacio íntimo de la

1 Un extenso debate sobre la articulación entre el análisis espacial y el análisis de redes sociales se puede revisar en el número especial (34(1)) de la revista Social Networks, del ańo 2012: Capturing Context: Integrating Spatial and Social Network Analyses. En https://www.sciencedirect.com/ journal/social-networks/vol/34/issue/ 1 
vivienda y la ciudad metropolitana, para lo cual son más relevantes aspectos funcionales como la conectividad y la disponibilidad de servicios, que su rol como espacio de contacto y encuentro con otros (Gravano, 2005; Link \& Méndez, 2011). Un tercer enfoque significativo para este trabajo es el llamado "efectos de barrio", a partir del cual se ha desarrollado una extensa línea de investigación sobre los factores a través de los cuales los barrios producen efectos sobre sus habitantes. Entre estos factores no solo se considera la composición sociodemográfica de los barrios, sino también los procesos sociales y los mecanismos institucionales presentes en dichos espacios, tales como el tipo de interacciones sociales, las actividades rutinarias de sus habitantes, la presencia de organizaciones e instituciones, y la eficacia de las normas sociales reflejadas en la confianza mutua y las expectativas compartidas (Sampson, Morenoff, Jeffrey, \& Gannon-Rowley, 2002).

Por otra parte, los vecindarios de vivienda social constituyen un caso específico de análisis que ha adquirido relevancia a partir de la creciente preocupación, a nivel global, por los procesos de fragmentación social de las áreas urbanas. Una de las preguntas que han abordado las investigaciones en este campo ha sido cómo este tipo de vecindarios incide en el debilitamiento o fortalecimiento de los vínculos comunitarios de sus habitantes (Hugman \& Sotiri, 2001). El supuesto que subyace a estos estudios es que las redes de capital social son un recurso potencial al que pueden recurrir las familias que viven en barrios de bajos ingresos para enfrentar ciertas necesidades y riesgos (Bashar \& Bramley, 2018).

Si bien es cierto que las particularidades de las políticas públicas de vivienda inciden en este análisis, la literatura de los países desarrollados ha identificado los mecanismos causales que vinculan las viviendas sociales con la formación de las redes de capital social. Primero, se identifican los efectos directos que generan los mismos programas de vivienda social, específicamente a través de la generación de un proceso de estigmatización, de las normas de arrendamiento y propiedad, o del diseńo físico del conjunto habitacional. En segundo lugar, se han identificado efectos indirectos a través de la acumulación de desventajas en los vecindarios de vivienda social, principalmente concentración de pobreza y desempleo, lo cual genera procesos de desafección respecto a las instituciones comunitarias, una reducción de las posibilidades de contacto entre los habitantes, y una merma de las relaciones entre ellos y las organizaciones sociales locales (Brisson \& Usher, 2005; Reingold, Van Ryzin, \& Ronda, 2001).

En el contexto latinoamericano, las investigaciones han mostrado que los vecindarios de vivienda social, generalmente caracterizados por una alta concentración de hogares pobres, se relacionan con una reducción de las oportunidades para la acumulación de capital social individual y colectivo. En este caso, la variable clave es la segregación residencial socioeconómica, la cual se asocia con una restricción de las redes de los pobres a personas de igual condición, y un debilitamiento de los vínculos con personas de diferente condición socioeconómica (Kaztman, 1999; Rodríguez \& Arriagada, 2004; Sabatini, 2006).

Las investigaciones en Chile sobre esta problemática plantean conclusiones divergentes, las cuales probablemente se explican por sus especificidades metodológicas y las particularidades de los casos estudiados. Por una parte, algunos estudios 
constatan, como característica de los conjuntos de vivienda social, la percepción generalizada de malestar y exclusión social, los bajos niveles de confianza interpersonal y la escasa participación en organizaciones locales (Segovia, 2005); por otra parte, algunas investigaciones refutan la hipótesis del "quiebre comunitario", mostrando una reconfiguración de los vínculos sociales y no necesariamente su agotamiento en los conjuntos de vivienda social (Tironi, 2003, 2009).

\section{Redefiniciones socioespaciales del capital social}

En este artículo se sigue una definición amplia del concepto de capital social, entendido como "la capacidad efectiva de movilizar productivamente y en beneficio del conjunto, los recursos asociativos que radican en las distintas redes sociales a las que tienen acceso los miembros del grupo en cuestión” (Atria, 2003, p. 583). Los recursos asociativos corresponden a las relaciones de confianza y reciprocidad entre los actores sociales, y a las normas e instituciones que facilitan las acciones de cooperación orientadas al logro de objetivos comunes (Durston, 2000).

Ahora, si bien la definición señalada entiende el capital social como un recurso colectivo o comunitario, no establece una relación específica entre dicho recurso y la dimensión espacial. En otras palabras, el capital social se asocia a un determinado grupo social, pero no se vincula con una localización espacial. Teniendo en cuenta ese vacío conceptual y empírico, en este artículo el análisis se focaliza en dos variantes del capital social que se asocian con el barrio en tanto escala espacial que puede incidir en la (re)producción de los recursos asociativos; a saber, el capital social vecinal y la eficacia colectiva. La distinción entre estos dos tipos de capital social permite diferenciar los tipos de vínculos sociales que se pueden configurar en el espacio barrial.

En primer lugar, el concepto de capital social vecinal implica una redefinición del nivel espacial en el que se miden las dimensiones del capital social: la confianza en los vecinos, la participación en organizaciones barriales, el apoyo social entre vecinos y la identificación o sentido de pertenencia que desarrollan hacia el barrio (Arriagada \& Sepúlveda, 2002). Por su parte, el concepto de eficacia colectiva se basa en la idea de que los barrios no solo son el sustento espacial de una red de relaciones sociales fuertes e íntimas, sino que también sirven de fundamento para la generación de confianzas y voluntades compartidas en torno a un bien común. Por lo tanto, la eficacia colectiva se define como la capacidad de los residentes de un barrio para lograr control social sobre su entorno y comprometerse con acciones colectivas en favor del bien común, sin necesidad de que existan lazos sociales fuertes (Morenoff, Sampson, \& Raudenbush, 2001). Según Sampson (2004), el concepto de eficacia colectiva se centra en la construcción de expectativas compartidas para la acción, lo cual se relaciona con la capacidad de los residentes de apropiarse del territorio y ejercer algún grado de control social sobre él.

En términos comparativos, el capital social vecinal se centra en la posibilidad de disponer de una red de relaciones sociales, mientras que la eficacia colectiva se focaliza en la activación de esa red para alcanzar objetivos comunes. Por lo tanto, las redes de capital social son una condición de la eficacia colectiva, pero no son 
suficientes para establecer una apropiación y un control social sobre el espacio que se habita (Sampson, 2004; Sampson, Morenoff, \& Earls, 2000).

\section{Factores socioespaciales que inciden sobre el capital social:}

concentración de pobreza, segregación residencial, y violencia y delincuencia

En este trabajo, las características de los barrios de vivienda social se han analizado considerando tres variables: la concentración de hogares pobres, la segregación residencial socioeconómica, y el nivel de violencia y delincuencia presente en el barrio. Estas variables se han seleccionado considerando su relevancia teórica y la disponibilidad de datos que permiten analizar empíricamente su efecto sobre la (re) producción del capital social a nivel de los conjuntos habitacionales.

En primer lugar, desde el enfoque de efectos de barrio se ha analizado cómo la concentración de pobreza en los barrios incide negativamente sobre las oportunidades de vida de las personas y la generación de redes de apoyo (Brisson \& Usher, 2007; Power \& Willmot, 2007; Small, 2004). Desde este enfoque, la relación entre pobreza y capital social está mediada por diferentes mecanismos y procesos sociales, tales como la desorganización y el aislamiento social, pero también por aspectos culturales y la capacidad de agencia de los propios sujetos que viven en los barrios (Small, 2004). En este sentido, es relevante el concepto de uso del espacio, el cual da cuenta de los procesos de construcción de una relación de identidad con el espacio que se habita (Fox Gotham, 2003). En esta misma línea, y centrándose en la experiencia de Chile, se ha planteado que para comprender la relación entre pobreza y capital social se deben considerar las transformaciones sociales de los sectores populares, reflejadas en que una gran parte de dichos sectores asimile los valores y cosmovisiones de la clase media, poniendo énfasis en sus proyectos individuales por sobre los proyectos colectivos (Salcedo, Sabatini, \& Rasse, 2009).

La segunda variable que se ha utilizado para caracterizar los vecindarios de vivienda social ha sido la segregación residencial socioeconómica, entendida como el grado de proximidad espacial o de aglomeración territorial de las familias pertenecientes a un mismo grupo socioeconómico (Sabatini, Cáceres, \& Cerda, 2001). En este ámbito, las investigaciones internacionales han mostrado que la homogeneidad socioeconómica y cultural (religión y etnia) de los barrios tiende a fomentar el capital social entre los vecinos, ya que consolida una identidad común y un sentido de pertenencia hacia ese entorno territorial que se comparte con otros iguales (Svendsen \& Haase, 2010). En el caso de Chile, la mayor parte de los estudios sobre los efectos de la segregación residencial socioeconómica se han focalizado en el capital social de aproximación; es decir, en el capital que se genera a partir de la vinculación con grupos sociales externos al barrio segregado. Al respecto, muestran que la segregación circunscribe las redes de los pobres a otras personas de igual condición, debilitando los vínculos y puentes entre clases sociales, y reproduciendo las desigualdades socioeconómicas que la generan (Arriagada, 2004; Rodríguez, 2001; Sabatini et al., 2001). Por otra parte, cuando se analiza la relación entre la segregación y la (re)producción de redes sociales entre los vecinos de un barrio, o capital social de vinculación, los resultados muestran un deterioro de la vida comunitaria del vecindario y una reducción de su capacidad de acción colectiva 
(Arriagada \& Rodríguez, 2003). Estos resultados también indican que tal relación se encuentra intermediada por una mayor presencia y percepción de situaciones de violencia y delincuencia en el barrio.

Teniendo en cuenta lo anterior, la tercera variable analizada ha sido el nivel de violencia y delincuencia presente en el barrio. Según señalan Moser y Mcllwaine (2009), una de las características de gran parte de las comunidades pobres urbanas de Latinoamérica es la existencia de "una compleja capa de múltiples formas de violencia y sobre todo la inseguridad y miedos relacionados, que se han vuelto 'rutinarios' o 'normales' dentro de la realidad de la vida diaria' (p. 12). Estos fenómenos no solo aparecen como un dato objetivo en las estadísticas policiales, sino que también se manifiestan en las percepciones subjetivas de los vecinos. Desde el enfoque de la exclusión social, se sostiene que "las causas que inciden sobre el incremento de la violencia y la criminalidad en este tipo de barrios refieren a la marginalidad, el abandono y aislamiento de grupos sociales en materia económica, social y cultural” (Lunecke, 2009, p. 42). Por lo tanto, la segregación residencial se asocia con la presencia de niveles más altos de violencia y criminalidad en los barrios. El problema de esta explicación causal de la violencia y la criminalidad es que resta importancia a las dinámicas sociales de cada barrio y a la capacidad de agencia de sus habitantes.

Las investigaciones realizadas en Chile muestran una relación negativa entre el nivel de violencia y delincuencia, por una parte, y la formación de capital social en barrios pobres, por la otra (Lunecke, 2009; Ruiz, 2009). La interrelación entre distintos tipos de violencia crea una red de miedo e inseguridad, lo que se asocia con menores niveles de confianza, el debilitamiento de las redes de apoyo y una escasa presencia de instituciones sociales locales, todo lo cual tiene como consecuencia un menoscabo del capital social (Ruiz, 2009). Por su parte, Lunecke (2009) plantea una distinción entre los efectos de la violencia y delincuencia a nivel individual y a nivel comunitario. A nivel individual, estos efectos se manifiestan en un alto grado de temor de los vecinos, lo cual impacta en sus prácticas cotidianas, generando un abandono de los espacios públicos, reclusión en el domicilio, deseos de cambiar de residencia y una pérdida del sentido de pertenencia hacia el barrio. En el nivel comunitario, el principal efecto que se identifica es la desconfianza interpersonal entre los vecinos. En el caso de los conjuntos de vivienda social, la percepción sobre los niveles de violencia y delincuencia en el barrio es una variable fundamental para entender las dinámicas de (re)producción del capital social, ya que "genera una sensación de pérdida del propio entorno y de desconfianza en la socialización con los otros [...] el temor inmoviliza para participar y asociarse, arraigándose un sistema de desconfianzas mutuas, cuyo principal contenido está dado por prejuicios, estereotipos y sospechas más o menos fundadas hacia los otros vecinos del barrio" (Lunecke, 2009, p. 40). 


\section{Metodología}

\section{Diseño de la investigación}

El diseño de esta investigación es no experimental, de corte transversal, de alcance correlacional-explicativo y de enfoque cuantitativo. Los datos analizados se obtuvieron a partir de la aplicación de una encuesta durante el año 2007, cuya población objetivo estuvo constituida por los jefes de hogar y dueñas de casa residentes en conjuntos de vivienda social de tres regiones de Chile: Metropolitana, Maule y Biobío. ${ }^{2}$ En total fueron encuestados 1.797 casos anidados en ocho conjuntos de vivienda social. La particularidad de esta muestra es que se seleccionaron conjuntos de vivienda social segregados y no segregados en términos socioeconómicos, pero lo más similares posibles en otras variables (tamaño, origen de los residentes, tipo de vivienda, antigüedad). La selección de los casos encuestados al interior de cada conjunto se realizó considerando como universo al total de las viviendas del conjunto respectivo. Los conjuntos con menos de 400 viviendas fueron censados, mientras que en los conjuntos con más de 400 viviendas los casos se seleccionaron a través de una muestra aleatoria simple con un $95 \%$ de confianza y un $5 \%$ de error muestral. En la siguiente tabla se presenta una descripción de la muestra (tabla 1).

\begin{tabular}{|l|l|l|c|l|}
\hline \multicolumn{1}{|c|}{ REGIÓN } & \multicolumn{1}{|c|}{ COMUNA } & \multicolumn{1}{c|}{$\begin{array}{c}\text { CONJUNTO DE } \\
\text { VIVIENDA SOCIAL }\end{array}$} & $\begin{array}{c}\text { CASOS } \\
\text { ENCUESTADOS }\end{array}$ & \multicolumn{1}{c|}{$\begin{array}{c}\text { SEGREGACIÓN } \\
\text { RESIDENCIAL }\end{array}$} \\
\hline \multirow{3}{*}{$\begin{array}{l}\text { Región } \\
\text { Metropolitana }\end{array}$} & Las Condes & El Bosque de la Villa & 303 & No segregado \\
\cline { 2 - 5 } & Maipú & San José & 236 & Segregado \\
\cline { 2 - 6 } & Puente Alto & Quitalmahue & 127 & Segregado \\
\hline \multirow{2}{*}{ Región del Maule } & Talca & Las Américas vi & 147 & Segregado \\
\cline { 2 - 6 } & San Luis viII & 193 & No segregado \\
\hline \multirow{3}{*}{ Región del Biobío } & San Pedro de la Paz & Michaihue & 311 & No segregado \\
\cline { 2 - 6 } & Talcahuano & Centinela & 251 & Segregado \\
\hline \multirow{2}{*}{ Total } & & & 229 & No segregado \\
\hline
\end{tabular}

TABLA I | Descripción de la muestra encuestada

FUENTE: ELABORACIÓN PROPIA

\section{Variables}

Las variables dependientes corresponden al capital social vecinal y la eficacia colectiva. El capital social vecinal se midió a partir de un índice sumatorio que incluye cuatro indicadores: confianza en vecinos, identificación y sentido de pertenencia con el conjunto habitacional, asociatividad dentro del conjunto habitacional y apoyo social vecinal. Por su parte, la eficacia colectiva se midió a partir de un índice

2 Para llevar a cabo este trabajo se utilizaron los datos de la encuesta aplicada en el marco del Proyecto Anillos "Barrios en crisis y barrios exitosos producidos por la política de vivienda social en Chile: influencia de la segregación residencial y lecciones de política” (Investigadores principales: Francisco Sabatini, Rodrigo Salcedo y Guillermo Wormald). 
sumatorio que incluye tres indicadores: la percepción del nivel de compromiso de los vecinos con acciones colectivas de bien común, la participación en acciones colectivas de bien común, y la actitud hacia el control social informal al interior del conjunto habitacional.

En cuanto a las variables independientes, se distinguen dos tipos. En primer lugar, las variables de caracterización individual de los encuestados (nivel 1), entre las que se incluyen las variables sociodemográficas (sexo, edad, nivel socioeconómico y nivel educacional), las variables de caracterización de acceso a la vivienda (tipo de asignación de la vivienda y modalidad de ocupación de la vivienda) y la percepción subjetiva de aislamiento social en el barrio. En segundo lugar, las dos variables de caracterización de los conjuntos habitacionales (nivel 2): la segregación residencial socioeconómica del conjunto habitacional (segregado y no segregado) y el índice promedio de percepción de violencia y delincuencia en el conjunto habitacional, el que resume la percepción de frecuencia de seis situaciones delictivas (tráfico de drogas, robo de casas, daño deliberado a bienes de uso común, consumo de drogas en lugares públicos, asaltos en las calles, acciones de violencia o balaceras).

\section{Análisis de datos}

El análisis estadístico de los datos contempló dos etapas. En la primera se realizaron análisis descriptivos y correlacionales, con el objeto de caracterizar a los sujetos encuestados y los conjuntos habitacionales. En la segunda etapa se estimaron los modelos de regresiones jerárquicas lineales, los cuales consideran la estructura anidada de los datos, es decir, el hecho de que los individuos encuestados fueron seleccionados en ocho conjuntos de vivienda social. La utilización de la regresión jerárquica o multinivel parte del supuesto estadístico de que los resultados de los individuos que viven en un mismo conjunto habitacional están correlacionados entre sí, lo cual viola el supuesto de la independencia de los errores en el cual se fundamenta el modelo de regresión lineal. Frente a este problema, la regresión multinivel modela las relaciones jerárquicas, proporcionando estimaciones sobre la variabilidad de los coeficientes de regresión a nivel de los conjuntos habitacionales (Oliver, Rosel, \& Jara, 2000).

\section{Resultados}

Para cada una de las variables dependientes se presentan los resultados descriptivos y un resumen de los resultados de los modelos de regresión jerárquica, los cuales se presentan en dos partes. Primero, los resultados de la prueba de ANOva (modelo restringido), que permite determinar si la pertenencia a un conjunto de vivienda social tiene un efecto significativo sobre las variables dependientes. En segundo lugar, los resultados de los coeficientes de regresión jerárquica, los cuales indican la fuerza y significancia de los efectos de las variables explicativas sobre la variable dependiente.

\section{Resultados del capital social vecinal}

El capital social vecinal se midió a través de un índice compuesto por cuatro dimensiones: confianza en vecinos; identificación y sentido de pertenencia con el conjunto 
habitacional; asociatividad al interior del conjunto habitacional; y percepción de apoyo social vecinal. Los resultados de cada dimensión son los siguientes:

- Confianza vecinal: el 57\% no confía en sus vecinos, el 33\% tiene poca confianza y solo el 10\% tiene mucha confianza.

- Identificación y sentido de pertenencia con el conjunto habitacional: el 33\% no se identifica, el $44 \%$ tiene una identificación intermedia y el $23 \%$, una alta identificación.

- Participación en organizaciones del barrio: el 71\% no participa en ninguna organización, el $24 \%$ participa en una organización y solo el $5 \%$ lo hace en dos o más organizaciones.

- Percepción de apoyo social vecinal (prestación de favores): el 36\% percibe un bajo nivel de apoyo vecinal, el 36\% un nivel intermedio y el $29 \%$, un nivel alto.

A partir de la sumatoria de los puntajes obtenidos en cada dimensión se calculó un puntaje total, cuyo rango va entre 4 y 12 puntos, donde 4 puntos es el menor nivel de capital social vecinal y 12 puntos el nivel más alto. En la muestra estudiada, el promedio general es de 6,74 puntos (desviación estándar=1,73). Los puntajes promedios varían significativamente entre los conjuntos habitacionales. El promedio más alto corresponde al conjunto El Bosque de la Villa $(7,26)$, mientras que el más bajo al conjunto habitacional San Luis viII $(5,73)$. Al recodificar el puntaje de este índice, ${ }^{3}$ se observa que el $47 \%$ de los encuestados se ubica en el nivel bajo, el $47 \%$ en el nivel medio y solo el 6\% en el nivel alto.

A continuación, se presentan los resultados de los modelos de regresión jerárquica:

a. Hipótesis nula. A partir del análisis de varianza (ANOvA) se testeó la hipótesis nula de que no existen diferencias estadísticamente significativas entre los promedios de cada conjunto habitacional en la variable capital social vecinal. Los resultados de este análisis $(\mathrm{F}=15,375$, sig. $=0,000)$ permiten rechazar esta hipótesis nula y concluir que las diferencias entre los promedios de los conjuntos habitacionales son estadísticamente significativas.

b. Coeficientes de regresión jerárquica. En primer lugar, se presentan los resultados de los modelos en los que se incluyen por separado las variables explicativas de nivel 1 y de nivel 2 . En el modelo 1 se incluyeron únicamente las variables explicativas de nivel 2 , de las cuales solo la variable percepción de violencia y delincuencia en el conjunto habitacional tiene un efecto significativo sobre el capital social vecinal: el aumento de una unidad en el índice de percepción de violencia y delincuencia disminuye en 0,22 puntos el puntaje en el índice de capital social vecinal, manteniendo constante la variable segregación residencial. En el modelo 2 se incorporaron las variables de caracterización sociodemográfica de los encuestados, de las cuales solo el sexo tiene un efecto significativo: el puntaje de los hombres en el índice de capital social vecinal supera al de las

3 La recodificación se realizó con los siguientes puntos de corte: nivel bajo=4-6 puntos, nivel medio=7-9 puntos, nivel alto $=10-12$ puntos. 
mujeres en 0,95 puntos. En los modelos 3 y 4 se incluyeron las variables de caracterización de acceso a la vivienda y la percepción subjetiva de aislamiento social. Se incluyen por separado el tipo de asignación de la vivienda (modelo 3) y la modalidad de ocupación de la vivienda (modelo 4), debido al alto nivel de asociación entre ellas: en un $81 \%$, los encuestados son al mismo tiempo asignatarios originales y propietarios de la vivienda. Los resultados indican que los asignatarios originales de la vivienda tienen un puntaje de capital social vecinal superior en 0,62 puntos al de los asignatarios posteriores, mientras que los propietarios de la vivienda tienen 0,54 puntos adicionales en comparación con los arrendatarios. Finalmente, en ambos modelos la percepción subjetiva de aislamiento social tiene un efecto significativo: el aumento en una unidad en el índice de percepción subjetiva de aislamiento social implica una disminución de 0,12 puntos en el índice de capital social vecinal (tabla 2).

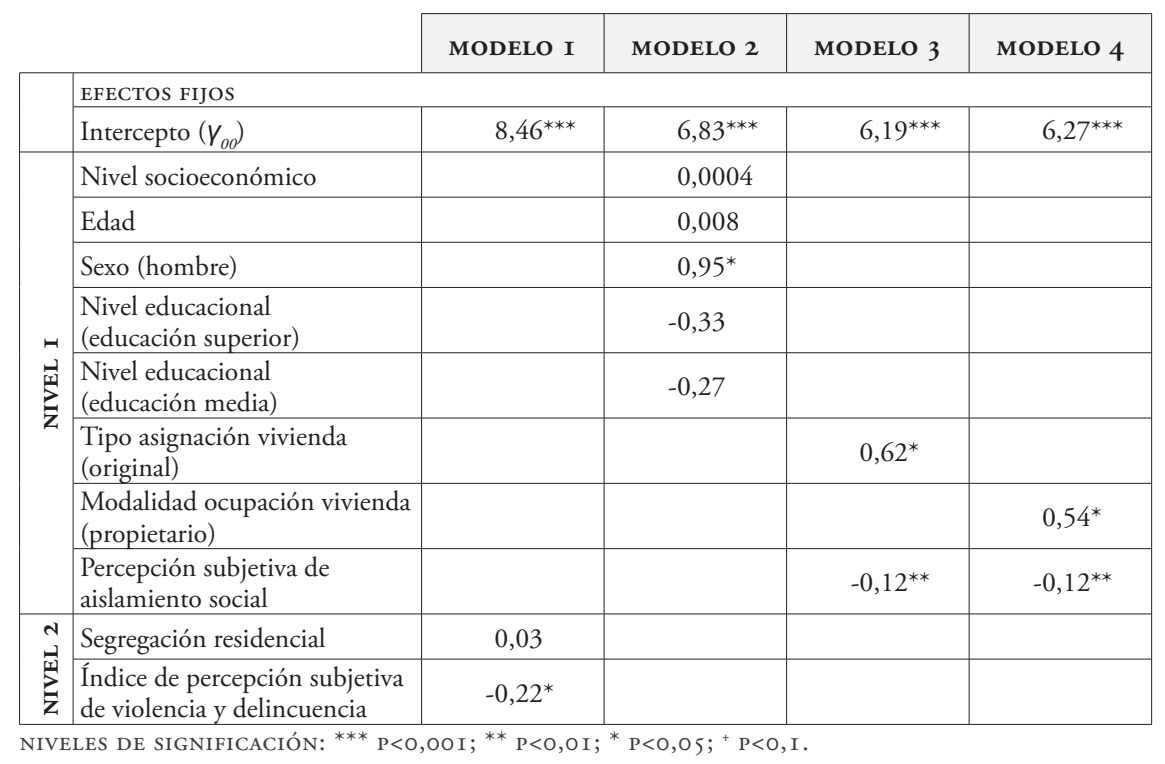

TABLA 2 | Resultados de regresiones jerárquicas de la variable capital social vecinal (Media como resultado y pendiente como resultado)

FUENTE: ELABORACIÓN PROPIA

A continuación se presentan los resultados de los cinco modelos de regresión jerárquica en los que se combinan los efectos de las variables explicativas de nivel 1 y 2. En el modelo 5 se incluyó la variable sexo, que es la única variable individual que tuvo un efecto directo significativo, y las dos variables de nivel 2: segregación residencial socioeconómica, y percepción de violencia y delincuencia. Los resultados indican que la variable sexo sigue teniendo un efecto significativo, a pesar del control de las variables de nivel 2: el puntaje de los hombres en el índice de capital social vecinal es 0,68 puntos más alto que el de las mujeres. Asimismo, se ratifica que la 
segregación residencial no tiene un efecto significativo, mientras que el aumento de una unidad en el índice de percepción de violencia y delincuencia tiene como efecto una disminución de 0,22 puntos en el índice de capital social vecinal.

En el modelo 6 se incluyeron dos variables de nivel 1 (tipo de asignación de la vivienda y percepción subjetiva de aislamiento social) y las dos variables de nivel 2. Los resultados indican que prácticamente ninguna de las variables incluidas en el modelo tiene un efecto significativo sobre el índice de capital social vecinal. Teniendo en cuenta el resultado anterior, en el modelo 7 se mantuvieron las mismas variables de nivel 1, pero solo se incluyó como variable de nivel 2 la percepción de violencia y delincuencia en el conjunto habitacional. En este modelo, las tres variables incluidas tienen un efecto significativo sobre el índice de capital social vecinal. Se ratifica así el efecto negativo de la percepción de violencia y delincuencia sobre la disposición de capital social vecinal: el aumento de una unidad en el índice de percepción de violencia y delincuencia disminuye en 0,14 puntos el puntaje en el índice de capital social vecinal. Por otra parte, se observa que el puntaje en el índice de capital social vecinal es 0,62 puntos más alto en los asignatarios originales de la vivienda en comparación con los asignatarios posteriores. En contrapartida, el aumento de un punto en el índice de percepción subjetiva de aislamiento social tiene como efecto una disminución de 0,11 puntos en el índice de capital social vecinal. Este último efecto negativo se ve potenciado con el aumento en la percepción de violencia y delincuencia: el efecto negativo de la percepción de aislamiento social aumenta en 0,05 puntos cuando el conjunto habitacional en el que vive el encuestado presenta una unidad adicional en el índice de percepción de violencia y delincuencia.

Finalmente, en los modelos 8 y 9 se repite el mismo análisis realizado en los dos modelos anteriores, con la diferencia de que se incluye la variable modalidad de ocupación de la vivienda en reemplazo del tipo de asignación de la vivienda. En el modelo en que se incluye la variable segregación residencial socioeconómica (modelo 8), prácticamente ninguna de las variables tiene un efecto significativo sobre el índice de capital social vecinal. Por su parte, en el modelo 9 solo las variables de nivel 1 tienen un efecto directo sobre el capital social vecinal: el aumento de un punto en el índice de percepción subjetiva de aislamiento social se asocia con una disminución de 0,11 puntos en el índice de capital social vecinal, mientras que el ser propietario de la vivienda implica un aumento de 0,55 puntos en el mismo índice. Sin embargo, este último efecto solo es significativo en los conjuntos no segregados, lo que significa que la segregación residencial socioeconómica anula el efecto positivo que tiene la propiedad de la vivienda sobre el capital social vecinal (tabla 3). 


\begin{tabular}{|c|c|c|c|c|c|}
\hline & MODELO 5 & MODELO 6 & MODELO 7 & MODELO 8 & MODELO 9 \\
\hline \multicolumn{6}{|l|}{ EFECTOS FIJOS } \\
\hline Intercepto $\left(\gamma_{o o}\right)$ & $6,71^{* * *}$ & $6,26^{* *}$ & $6,2^{* * *}$ & $6,37^{* * *}$ & $6,2^{* * *}$ \\
\hline Segregación residencial & $-0,009$ & $-0,12$ & - & $-0,25$ & - \\
\hline $\begin{array}{l}\text { Índice de percepción subjetiva de } \\
\text { violencia y delincuencia }\end{array}$ & $-0,22^{*}$ & $-0,13$ & $-0,14^{*}$ & $-0,11$ & $-0,13$ \\
\hline \multicolumn{6}{|l|}{ Sexo (hombre) slope } \\
\hline Intercepto & $0,68^{*}$ & - & - & - & - \\
\hline Segregación residencial & 0,75 & - & - & - & - \\
\hline $\begin{array}{l}\text { Índice de percepción subjetiva de } \\
\text { violencia y delincuencia }\end{array}$ & $-0,03$ & - & - & - & - \\
\hline \multicolumn{6}{|c|}{ Tipo de asignatario de la vivienda (original) slope } \\
\hline Intercepto & - & 0,51 & $0,62^{*}$ & - & - \\
\hline Segregación residencial & - & 0,21 & - & - & - \\
\hline $\begin{array}{l}\text { Índice de percepción subjetiva de } \\
\text { violencia y delincuencia }\end{array}$ & - & $-0,1$ & $-0,09$ & - & - \\
\hline \multicolumn{6}{|c|}{ Percepción subjetiva de aislamiento social slope } \\
\hline Intercepto & - & $-0,08$ & $-0,11^{* *}$ & $-0,08$ & $-0,11^{* *}$ \\
\hline Segregación residencial & - & $-0,06$ & - & $-0,06$ & - \\
\hline $\begin{array}{l}\text { Índice de percepción subjetiva de } \\
\text { violencia y delincuencia }\end{array}$ & - & $-0,05+$ & $-0,05^{* *}$ & $-0,05+$ & $-0,05^{* *}$ \\
\hline \multicolumn{6}{|c|}{ Modalidad de ocupación de la vivienda (propietario) slope } \\
\hline Intercepto & - & - & - & 0,41 & $0,55^{*}$ \\
\hline Segregación residencial & - & - & - & 0,33 & - \\
\hline $\begin{array}{l}\text { Índice de percepción subjetiva de } \\
\text { violencia y delincuencia }\end{array}$ & - & - & - & $-0,12$ & $-0,1$ \\
\hline
\end{tabular}

\section{TABLA 3 Resultados de regresiones jerárquicas de la variable capital social vecinal. Modelo intercepto y pendiente como resultados}

FUENTE: ELABORACIÓN PROPIA

\section{Resultados de la eficacia colectiva}

Para medir la eficacia colectiva se utilizó un índice compuesto por tres dimensiones: percepción de compromiso vecinal con acciones colectivas de bien común; participación en acciones colectivas de bien común; y actitud hacia el control social informal. Los resultados de cada dimensión son los siguientes:

- Percepción del nivel de compromiso vecinal con acciones colectivas: el $22 \%$ percibe un nivel bajo de compromiso, el $28 \%$ un nivel intermedio y el $50 \%$, un nivel de alto. 
- Participación en acciones colectivas: el $74 \%$ tiene un bajo nivel de participación (máximo una acción), el 12\% un nivel intermedio (2-3 acciones) y el 14\%, un nivel alto (4-6 acciones).

- Actitud hacia el control social informal: el 33\% presenta una actitud negativa, el $42 \%$ una actitud intermedia, y el $26 \%$, una actitud positiva.

A partir de la sumatoria de los puntajes obtenidos en estas tres dimensiones se calculó un puntaje total cuyo rango de valores va entre 3 y 9 puntos, en donde 3 representa el nivel más bajo de eficacia colectiva y 9 puntos el nivel más alto. En la muestra estudiada, el promedio general es de 5,67 puntos (desviación estándar=1,5). El promedio más alto corresponde al conjunto Centinela $(6,26)$, mientras que el más bajo al conjunto habitacional San Luis viII $(4,88)$. Al recodificar el puntaje de este índice, ${ }^{4}$ el resultado es que el $23 \%$ de los encuestados se ubica en el nivel bajo, el $48 \%$ en el nivel medio y el $29 \%$, en el nivel alto.

A continuación, se presentan los resultados de los modelos de regresión jerárquica (tabla 4):

a. Hipótesis nula. A partir del análisis de varianza (ANOvA), se sometió a prueba la hipótesis de que no existen diferencias estadísticamente significativas entre los promedios de cada conjunto habitacional en la variable eficacia colectiva. Los resultados de este análisis $(\mathrm{F}=17,375$, sig. $=0,000)$ permiten rechazar esta hipótesis nula y concluir que los promedios en el índice de eficacia colectiva difieren significativamente entre cada conjunto de vivienda social, lo cual justifica la estimación de los modelos de regresión multinivel.

b. Coeficientes de regresión jerárquica. En primer lugar, se presentan los resultados de los modelos en los que se incluyen por separado las variables explicativas de nivel 1 y las variables de nivel 2. En el modelo 1 se incluyen únicamente las variables explicativas de nivel 2, de las cuales solo la percepción de violencia y delincuencia en el conjunto habitacional tiene un efecto significativo sobre el índice de eficacia colectiva: el aumento de una unidad en el índice de percepción de violencia y delincuencia disminuye en 0,2 puntos el puntaje en el índice de eficacia colectiva, manteniendo constante el resto de las variables. En el modelo 2 se incorporaron las variables de caracterización sociodemográfica de los encuestados, de las cuales el nivel socioeconómico y la edad tienen un efecto bajo pero significativo sobre el índice de eficacia colectiva. En los modelos 3 y 4 se incluyeron las variables de caracterización de acceso a la vivienda y la percepción subjetiva de aislamiento social, incorporando por separado el tipo de asignación de la vivienda (modelo 3) y la modalidad de ocupación de la vivienda (modelo 4). Los resultados del modelo 3 muestran que tanto el tipo de asignación de la vivienda como la percepción subjetiva de aislamiento social tienen un efecto significativo sobre el índice de eficacia colectiva: los asignatarios originales de la vivienda presentan un puntaje 0,5 puntos más alto, mientras que el aumento en una unidad en el índice de percepción subjetiva de aislamiento social implica 
una disminución de 0,07 puntos en el índice de eficacia colectiva. Por otra parte, el modelo 4 muestra que la modalidad de ocupación también tiene un efecto significativo: los propietarios de la vivienda presentan 0,45 puntos adicionales en el índice de eficacia colectiva. Estos resultados muestran que, en un contexto de postulación individual a la vivienda social, las experiencias de ser propietario y de vivir desde el comienzo en el conjunto habitacional contribuyen a afianzar el compromiso vecinal con el bienestar colectivo del barrio.

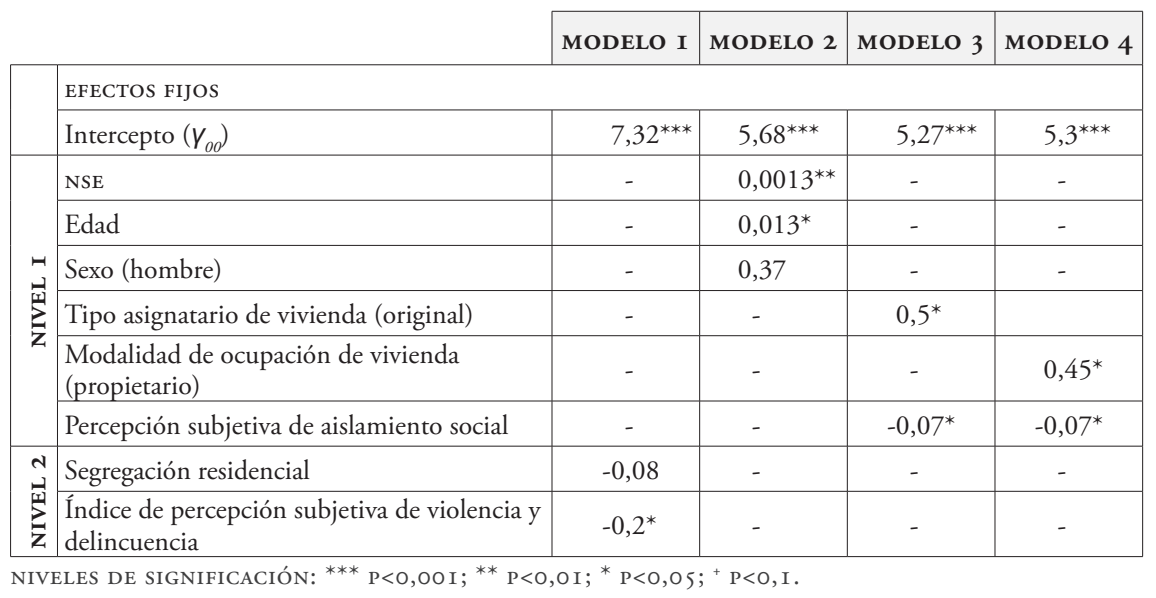

TABLA 4 Resultados de regresiones jerárquicas de la variable eficacia colectiva. Modelos media como resultado y pendiente como resultado FUENTE: ELABORACIÓN PROPIA

A continuación, se presentan los resultados de los modelos de regresión jerárquica en los que se combinan los efectos de las variables explicativas de nivel 1 y 2 . En el modelo 5 se incluyeron las dos variables de caracterización socioeconómica que tuvieron efectos significativos en el modelo 2 (nivel socioeconómico y edad), junto con las variables segregación residencial socioeconómica, y percepción de violencia y delincuencia en el conjunto habitacional. Los resultados muestran que cuando se incluyen las variables de nivel 2, el nivel socioeconómico y la edad dejan de tener un efecto significativo sobre el índice de eficacia colectiva. Por otra parte, se ratifica que solo la percepción de violencia y delincuencia tiene un efecto significativo sobre la eficacia colectiva: el aumento de una unidad en el índice de percepción de violencia y delincuencia tiene como efecto una disminución de 0,2 puntos en el índice de eficacia colectiva.

En el modelo 6 se incluyeron dos variables que caracterizan las condiciones de acceso a la vivienda (tipo de asignación de la vivienda y percepción subjetiva de aislamiento social) y las dos variables de nivel 2. En este modelo solo la percepción de violencia y delincuencia tiene un efecto significativo sobre el índice de eficacia colectiva: el aumento de una unidad en el índice de percepción de violencia y delincuencia tiene como efecto una disminución de 0,32 puntos en el índice de eficacia colectiva. 
Sin embargo, cuando se excluye de este análisis la variable segregación residencial socioeconómica (modelo 7), el resultado es que todas las variables tienen un efecto significativo sobre el índice de eficacia colectiva: el aumento de una unidad en el índice de percepción de violencia y delincuencia tiene como efecto una disminución de 0,35 puntos en el índice de eficacia colectiva; los asignatarios originales de la vivienda tienen 0,45 puntos adicionales en el índice de eficacia colectiva; y el aumento de una unidad en el índice de percepción subjetiva de aislamiento social tiene como efecto una disminución de 0,07 puntos en el índice de eficacia colectiva, disminución que se ve potenciada en 0,03 puntos cuando aumenta en una unidad el puntaje en el índice de percepción de violencia y delincuencia en el conjunto habitacional.

En el modelo 8 se incluyeron nuevamente las dos variables de nivel 2 junto con las variables modalidad de ocupación de la vivienda y percepción subjetiva de aislamiento social. Al igual que en el modelo 6, los resultados de este modelo indican que solo la percepción de violencia y delincuencia en el conjunto habitacional tiene un efecto significativo sobre el índice de eficacia colectiva: el aumento de una unidad en el índice de percepción de violencia y delincuencia tiene como efecto una disminución de 0,31 puntos en el índice de eficacia colectiva. Por otra parte, cuando se excluye de este análisis la variable segregación residencial socioeconómica (modelo 9), las tres variables incluidas tienen un efecto significativo sobre el índice de eficacia colectiva: el aumento de una unidad en el índice de percepción de violencia y delincuencia tiene como efecto una disminución de 0,34 puntos en el índice de eficacia colectiva; el hecho de ser propietario de la vivienda implica un aumento de 0,41 puntos en el índice de eficacia colectiva, efecto que se ve potenciado en 0,14 puntos con el aumento en una unidad del puntaje en el índice de percepción de violencia y delincuencia en el conjunto habitacional; y el aumento de una unidad en el índice de percepción subjetiva de aislamiento social se asocia con una disminución de 0,06 puntos en el índice de eficacia colectiva (tabla 5).

\begin{tabular}{|c|c|c|c|c|c|}
\hline & MODELO 5 & MODELO 6 & MODELO 7 & MODELO 8 & MODELO 9 \\
\hline \multicolumn{6}{|l|}{ EFECTOS FIJOS } \\
\hline Intercepto $\left(\gamma_{o o}\right)$ & - & - & - & - & - \\
\hline Intercepto & $7,28^{* * *}$ & $5,52^{* * *}$ & $5,32^{* * *}$ & $5,57^{* * *}$ & $5,36^{* * *}$ \\
\hline Segregación residencial & $-0,08$ & $-0,45$ & & $-0,52$ & - \\
\hline $\begin{array}{l}\text { Índice de percepción subjetiva de } \\
\text { violencia y delincuencia }\end{array}$ & $-0,2+$ & $-0,32^{*}$ & $-0,35^{* * *}$ & $-0,31^{*}$ & $-0,34^{* * *}$ \\
\hline \multicolumn{6}{|l|}{ Nivel socioeconómico slope } \\
\hline Intercepto & 0,003 & - & - & - & - \\
\hline Segregación residencial & 0,0004 & - & - & - & - \\
\hline $\begin{array}{l}\text { Índice de percepción subjetiva de } \\
\text { violencia y delincuencia }\end{array}$ & $-0,0002$ & - & - & - & - \\
\hline
\end{tabular}


(continuación)

\begin{tabular}{|c|c|c|c|c|c|}
\hline & MODELO 5 & MODELO 6 & MODELO 7 & MODELO 8 & MODELO 9 \\
\hline Intercepto & 0,05 & - & - & - & - \\
\hline Segregación residencial & 0,0083 & - & - & - & - \\
\hline $\begin{array}{l}\text { Índice de percepción subjetiva de } \\
\text { violencia y delincuencia }\end{array}$ & $-0,005$ & - & - & - & - \\
\hline \multicolumn{6}{|c|}{ Tipo de asignatario de la vivienda (original) slope } \\
\hline Intercepto & - & 0,26 & $0,45^{*}$ & - & - \\
\hline Segregación residencial & - & 0,43 & & - & - \\
\hline $\begin{array}{l}\text { Índice de percepción subjetiva de } \\
\text { violencia y delincuencia }\end{array}$ & - & 0,13 & 0,16 & & - \\
\hline \multicolumn{6}{|c|}{ Percepción subjetiva de aislamiento social slope } \\
\hline Intercepto & - & $-0,06$ & $-0,07^{*}$ & $-0,05$ & $-0,06^{*}$ \\
\hline Segregación residencial & - & $-0,01$ & - & $-0,01$ & \\
\hline $\begin{array}{l}\text { Índice de percepción subjetiva de } \\
\text { violencia y delincuencia }\end{array}$ & - & $-0,03$ & $-0,03^{*}$ & $-0,03$ & $-0,03$ \\
\hline \multicolumn{6}{|c|}{ Modalidad de ocupación de la vivienda (propietario) slope } \\
\hline Intercepto & - & - & - & 0,21 & $0,41^{*}$ \\
\hline Segregación residencial & - & - & - & 0,48 & \\
\hline $\begin{array}{l}\text { Índice de percepción subjetiva de } \\
\text { violencia y delincuencia }\end{array}$ & - & - & - & 0,11 & $0,14^{*}$ \\
\hline
\end{tabular}

NIVELES DE SIGNIFICACIÓN: ${ }^{* * *} \mathrm{P}<\mathrm{O}, \mathrm{OOI} ;{ }^{* *} \mathrm{P}<\mathrm{O}, \mathrm{OI} ;{ }^{*} \mathrm{P}<\mathrm{O}, 05 ;{ }^{+} \mathrm{P}<\mathrm{O}, \mathrm{I}$.

Tabla 5 Resultados de regresiones jerárquicas de la variable eficacia colectiva.

Modelo intercepto y pendiente como resultados

FUENTE: ELABORACIÓN PROPIA

\section{Discusión de resultados}

Los resultados de este trabajo resaltan cuatro características del capital social en los conjuntos de vivienda social en Chile, referidas al stock acumulado de capital social, a las formas de acceso a la vivienda, a las dinámicas sociales al interior de los barrios, y al impacto de la segregación socioeconómica.

\section{Las características del stock acumulado de capital social}

Según los resultados del estudio, el stock acumulado de capital social en los barrios analizados presenta tres características diferenciadoras. En primer lugar, los vínculos sociales se construyen sobre la base de bajos niveles de confianza vecinal, lo cual indica que la generación de vínculos vecinales resulta altamente riesgosa, en el sentido de que se instala la expectativa de que los otros pueden comportarse oportunistamente o bien no cumplir con aquello a lo cual se han comprometido (Nootebom, 2007). En segundo lugar, las redes de capital social se generan fundamentalmente a partir de relaciones de apoyo social circunstancial (prestación de favores) y no se basan en acciones asociativas o colaborativas. Finalmente, las redes de eficacia colectiva se basan más en la predisposición al control social informal que en prácticas cooperativas entre los vecinos. Esto indica que si bien existe una predisposición positiva hacia la posibilidad de comprometerse activamente en lo que sucede en el 
conjunto habitacional, ella no se expresa necesariamente en prácticas efectivas de cooperación que tengan un beneficio colectivo.

Las formas de acceso a la vivienda y su impacto sobre la apropiación territorial Uno de los resultados más interesantes es que el ser asignatario original y propietario de la vivienda son factores que inciden positivamente en la generación de vínculos sociales basados en la confianza y de apoyo social recíproco entre vecinos, así como en una gestión colectiva de los espacios comunes.

El ser el asignatario original de la vivienda da cuenta de un vínculo histórico con el conjunto habitacional, el cual en la mayoría de los casos constituye un punto de inflexión para quienes acceden a la vivienda social, ya que representa el paso desde el campamento o allegamiento a la vivienda propia. En este sentido, el haber estado presente en el hito fundacional de la comunidad contribuye a la creación de un nosotros y de un sentido de pertenencia al barrio. Por otra parte, el ser propietario de la vivienda es un eje articulador de las relaciones vecinales (Skewes, 2005), ya que se asocia con un mayor grado de interés por el desarrollo de los espacios colectivos y con la construcción de un sentido de pertenencia y de corresponsabilidad hacia el barrio. Sin embargo, en los conjuntos de vivienda social segregados estas variables no se ven reflejadas en mayores niveles de capital social vecinal y eficacia colectiva. La segregación, por lo tanto, es un obstáculo para que estas modalidades de acceso a la vivienda se expresen en un sentido de apropiación, de identificación y de implicación con el barrio.

\section{Las dinámicas sociales al interior de los barrios:}

\section{el impacto negativo de la violencia y la delincuencia}

Los resultados de esta investigación muestran que el nivel de violencia y delincuencia que los vecinos perciben en sus barrios se asocia negativamente con la producción de redes de capital social. Este efecto negativo se puede interpretar desde dos perspectivas. En primer lugar, las situaciones de violencia y delincuencia generan un contexto social de temor, inseguridad y desconfianza en el otro, el que dificulta la generación de redes de apoyo social basadas en la reciprocidad entre vecinos y la posibilidad de que la propia comunidad establezca pautas normativas comunes y desarrollen acciones colectivas que beneficien al barrio. Así, el debilitamiento de la confianza entre vecinos produce un efecto de retraimiento de los vínculos sociales hacia las redes centradas en el círculo más íntimo y familiar (Moser \& McIlwaine, 2009). Se configura un círculo vicioso entre las situaciones de violencia y delincuencia, por una parte, y la desconfianza y ausencia de redes sociales entre los vecinos, que potencialmente permitirían enfrentar colectivamente estas situaciones, por la otra. La segunda interpretación se centra en los efectos negativos que las situaciones de violencia y delincuencia generan en la relación entre los individuos y el espacio habitado. En la medida en que los hechos de violencia y delincuencia forman parte de la cotidianeidad del barrio, se tiende a generar una desafección hacia el lugar que se habita, lo que repercute en menores niveles de implicación y participación en acciones colectivas que beneficien a la comunidad, dificultando a 
su vez la generación de normas sociales que permitan ejercer un control social sobre este espacio (Lunecke, 2009; Vidal \& Pol, 2005).

\section{El impacto de la segregación residencial socioeconómica:}

la distinción entre la dimensión estructural y la dimensión subjetiva

Para analizar el efecto de la segregación residencial socioeconómica, es fundamental diferenciar entre su dimensión estructural y su expresión en la subjetividad y experiencias cotidianas de los sujetos. Los resultados presentados refutan las explicaciones que plantean que la segregación residencial tiene un efecto significativo, ya sea positivo o negativo, sobre la (re)producción del capital social. Sin embargo, la segregación residencial sí tiene un efecto negativo sobre la identificación y el sentido de pertenencia hacia el barrio. Por otra parte, la expresión subjetiva de la segregación residencial, medida como la percepción de aislamiento social frente a determinados servicios públicos, tiene un efecto negativo sobre la formación de redes de capital social entre los vecinos. Esta expresión subjetiva de la segregación residencial funciona como un mecanismo de intermediación entre la segregación estructural y los procesos de desintegración social que experimentan los barrios, ya que debilita el vínculo subjetivo que los residentes establecen con los espacios que habitan.

\section{Conclusión}

El objetivo principal de este artículo ha sido analizar las redes de capital social a nivel de la escala local de los conjuntos de vivienda social, explicitando una diferenciación entre distintos tipos de redes a partir de los conceptos de capital social vecinal y eficacia colectiva. Con base en los resultados presentados, se puede concluir que en los barrios de vivienda social predomina un tipo de vínculo social circunstancial y motivado por objetivos comunes concretos, lo cual representa un quiebre con la idea del barrio como espacio de relaciones de confianza y de una asociatividad comprometida. En relación con la dimensión espacial del capital social, se puede concluir que, en el caso de Chile, las variables relevantes son aquellas que caracterizan las dinámicas sociales al interior de los barrios, principalmente la percepción de la violencia y delincuencia, y aquellas que definen la identificación subjetiva con el conjunto habitacional. Por otra parte, los datos muestran que la segregación residencial socioeconómica no es relevante por sí misma para explicar los niveles de redes sociales existentes en los barrios.

Estos resultados son relevantes, ya que implican una revisión conceptual y empírica del espacio urbano, y principalmente de los barrios de vivienda social, como un factor que puede limitar o potenciar la formación de redes sociales. En este sentido, el espacio urbano no se puede analizar únicamente desde la estructura socioterritorial, entendida como la separación de grupos socioeconómicos en el espacio urbano. También se deben considerar aquellos factores que dan cuenta de las prácticas sociales que los vecinos desarrollan en los barrios y el modo en que se construye un sentido de pertenencia y de identificación con el espacio que se habita, lo cual está relacionado con los aspectos funcionales del barrio (conectividad y acceso a servicios). 
Asimismo, la distinción conceptual y empírica entre el capital social vecinal y la eficacia colectiva -distinción que se propone en este artículo- contribuye a matizar la hipótesis del quiebre comunitario, la cual tiende a dicotomizar el análisis de las redes sociales al interior de los barrios de vivienda social, y a profundizar en el análisis del capital social de vinculación (bonding social capital). En este sentido, los resultados presentados dan cuenta de que las dinámicas de formación de redes sociales al interior de los barrios de vivienda social son complejas, ya que conjugan simultáneamente elementos propios de los vínculos sociales fuertes y débiles.

Finalmente, los resultados de este trabajo aportan elementos relevantes para analizar las políticas públicas que se han desarrollado para revertir la situación de deterioro físico y social de determinados barrios. Por una parte, se resalta la importancia de los factores asociados a la identidad con el barrio y a la seguridad; pero, al mismo tiempo, se destaca cómo la fuerte tendencia a la formación de vínculos sociales esporádicos y circunstanciales plantea el desafío de la institucionalización y la sostenibilidad de dichas intervenciones estatales, esto es, de las políticas relativas a la vivienda y el hábitat urbano.

\section{Referencias bibliográficas}

Abbott, A. (1997). Of time and space: the contemporary relevance of the Chicago School. Social Forces 75, 1149-1182. http://doi.org/10.2307/2580667

Adams, J., Faust, K., \& Lovasi, G. (2012). Introduction to the special issue on Network Dynamics (Part 2). Social Networks, 34(3), 289-290. http://doi.org/10.1016/j. socnet.2009.12.002

Arriagada, C. (2004). Determinantes de gran escala de la segregación residencial del área metropolitana del Gran Santiago y efectos espaciales locales en comunidades pobres. En G. Cáceres \& F. Sabatini (eds.), Barrios cerrados en Santiago de Chile: entre la exclusión y la integración social. Santiago de Chile: Lincoln Institute of Land Policy/ Instituto de Geografía, P. Universidad Católica de Chile.

Arriagada, C. \& Rodríguez, J. (2003). Segregación residencial en áreas metropolitanas de América Latina: magnitud, caracteristicas, evolución e implicaciones de política. Santiago de Chile: Comisión Económica para América Latina y el Caribe (Cepal). https://repositorio. cepal.org/bitstream/handle/11362/7189/1/S0310709_es.pdf

Arriagada, C. \& Sepúlveda, D. (2002). Satisfacción residencial en la vivienda básica Serviu: la perspectiva del capital social. Santiago de Chile: Ministerio de Vivienda y Urbanismo (Minvu). http://www.minvu.cl/incjs/download.aspx?glb_cod_ nodo $=20070411164455 \%$ hdd_nom_archivo=Satisfacci $\%$ C3\%B3n \% 20 Residencial\%20Capital\%20Social.pdf

Atria, R. (2003). Capital social: concepto, dimensiones y estrategias para su desarrollo. En R. Atria, M. Siles, I. Arriagada, L. J. Robinson, \& S. Whiterford (eds.), Capital social y reducción de la pobreza en América Latina y el Caribe: en busca de un nuevo paradigma (pp. 581-590). Santiago de Chile: Comisión Económica para América Latina y el Caribe (Cepal). https://repositorio.cepal.org/handle/11362/2345 
Bashar, T. \& Bramley, G. (2018). Social capital and neighborhood cooperation: Implications for development of the urban poor in LDCs. Urban Studies. https://doi. org/10.1177/0042098018797945

Blokland, T. \& Savage, M. (eds.) (2008). Networked urbanism: social capital in the city. Burlington, vт: Ashgate.

Brisson, D. \& Usher, C. L. (2005). Bonding social capital in low-income neighborhoods. Family Relations, 54, 644-653. https://doi.org/10.1111/j.1741-3729.2005.00348.x

Brisson, D. \& Usher, C. L. (2007). The effects of informal neighborhood bonding social capital and neighborhood context on homeownership for families living in poverty. Journal of Urban Affairs, 29(1), 65-75. https://doi.org/10.1111/j.1467-9906.2007.00323.x

Ducci, M. E. (2005). La política de vivienda como instrumento de desintegración social. Efectos de una política de vivienda exitosa. En M. J. Castillo \& R. Hidalgo (eds.), 1906/2006 Cien años de políticas de vivienda en Chile. Santiago de Chile: Universidad de Chile.

Durston, J. (2000). ¿Qué es el capital social comunitario? Santiago de Chile: Comisión Económica para América Latina y el Caribe (Cepal). https://repositorio.cepal.org/ bitstream/handle/11362/5969/S0007574_es.pdf

Forrest, R. \& Kearns, A. (2001). Social cohesion, social capital and the neighbourhood. Urban Studies, 38(12), 2125-2143. https://doi.org/10.1080/00420980120087081

Fox Gotham, K. (2003). Using space: agency and identity in a public-housing development. International Journal of Urban and Regional Research, 27(3), 723-737. https://doi. org/10.1111/1540-6040.00023

Gravano, A. (2005). El barrio en la teoría social. Buenos Aires: Espacio.

Häkli, J. \& Minca, C. (eds.) (2009). Social capital and urban networks of trust. Aldershot, uk: Ashgate.

Hidalgo, R. (2004). La vivienda social en Santiago de Chile en la segunda mitad del siglo xx: Actores relevantes y tendencias espaciales. En C. De Mattos, M. E. Ducci, A. Rodríguez, \& G. Yáńez (eds.), Santiago en la globalización, ¿una nueva ciudad? (pp. 219-241). Santiago de Chile: Ediciones SUR. http://www.sitiosur.cl/detalle-dela-publicacion/?santiago-en-la-globalizacion-una-nueva-ciudad\#descargar

Hugman, R. \& Sotiri, M. (2001). Housing, social capital and stronger communities. Australian Housing and Urban Research Institute. https://www.researchgate.net/profile/Richard_ Hugman/publication/277295103_authored_by/links/55c01b8908 ae092e9666a68b. pdf

Kaztman, R. (1999). El vecindario también importa. En R. Kaztman (ed.), Activos y estructuras de oportunidades. Estudios sobre las raíces de la vulnerabilidad social en Uruguay (pp. 263307). Montevideo: Comisión Económica para América Latina y el Caribe (Cepal). https://www.cepal.org/cgi-bin/getProd.asp?xml=/publicaciones/xml/2/10772/ P10772.xml

Link, F. \& Méndez, M. L. (2011). Negociando identidad. Las posibilidades del barrio como espacio vinculante con la Gran Ciudad. En M. Cea (coord.), Chile 2010. Percepciones $y$ actitudes sociales (pp. 137-148). Santiago de Chile: Facultad de Ciencias Sociales e Historia ( $\mathrm{FSCH}$ )/Instituto de Investigación en Ciencias Sociales (ICSO), Universidad Diego Portales. http://encuesta.udp.cl/descargas/publicaciones/2010/Chile\%202010 $\% 20$ Percepciones $\% 20 y \% 20$ Actitudes $\% 20$ Sociales.pdf 
Lunecke, A. (2009). Exclusión social, tráfico de drogas y vulnerabilidad barrial. En A. Lunecke, A. M. Munizaga, \& J. C. Ruiz (eds.), Violencia y delincuencia en barrios: sistematización de experiencias (pp. 40-52). Santiago de Chile: Fundación Paz Ciudadana/Universidad Alberto Hurtado. https://pazciudadana.cl/biblioteca/documentos/violencia-y-delin cuencia-en-barrios-sistematizacion-de-experiencias/

Lunecke, A. (2012). Violencia urbana, exclusión social y procesos de guetización: La trayectoria de la población Santa Adriana. Revista INVI, 27(74), 287-313. https://doi. org/10.4067/S0718-83582012000100009

Morenoff, J. D., Sampson, R. J., \& Raudenbush, S. W. (2001). Neighborhood inequality, collective efficacy, and the spatial dynamics of urban violence. Criminology, 39(3), 517-560. https://doi.org/10.1111/j.1745-9125.2001.tb00932.x

Moser, C. \& Mcllwaine, C. (2009). La violencia urbana en Latinoamérica como problema de desarrollo: hacia un marco para reducir la violencia. En A. Lunecke, A. M. Munizaga, \& J. C. Ruiz (eds.), Violencia y delincuencia en barrios: sistematización de experiencias (pp. 12-39). Santiago de Chile: Fundación Paz Ciudadana/Universidad Alberto Hurtado. https://pazciudadana.cl/biblioteca/documentos/violencia-y-delincuencia-en -barrios-sistematizacion-de-experiencias/

Nootebom, B. (2007). Social capital, institutions and trust. Review of Social Economy, 65(1), 29-53. https://doi.org/10.1080/00346760601132154

Oliver, J. C., Rosel, J., \& Jara, P. (2000). Modelos de regresión multinivel: aplicación en psicología escolar. Psicothema, 12(3), 487-494. http://www.psicothema.com/pdf/361. pdf

Power, A. \& Willmot, H. (2007). Social capital within the neighbourhood. London: Center for Analysis of Social Exclusion. http://sticerd.lse.ac.uk/dps/case/cr/CASEreport38.pdf

Reingold, D., Van Ryzin, G., \& Ronda, M. (2001). Does urban public housing diminish the social capital and labor force activity of its tenants? Journal of Policy Analysis and Management, 20(3), 485-504. https://doi.org/10.1002/pam.1004

Rodríguez, J. (2001). Segregación residencial socioeconómica: ¿qué es?, ¿cómo se mide?, ¿qué está pasando?, ¿importa? Santiago de Chile: Comisión Económica para América Latina y el Caribe (Cepal). https://www.cepal.org/es/publicaciones/7149-segregacion-residencialsocioeconomica-que-es-como-se-mide-que-esta-pasando

Rodríguez, J. \& Arriagada, C. (2004). Segregación residencial en la ciudad latinoamericana. Revista EURE, 29(89), 5-24. http://doi.org/10.4067/S0250-71612004008900001

Ruiz, J. C. (2009). Violencia y capital social en Santiago: Notas para entender los barrios vulnerados y los barrios críticos. En A. Lunecke, A. M. Munizaga, \& J. C. Ruiz (eds.), Violencia y delincuencia en barrios: sistematización de experiencias (pp. 5366). Santiago de Chile: Fundación Paz Ciudadana/Universidad Alberto Hurtado. https://pazciudadana.cl/biblioteca/documentos/violencia-y-delincuencia-en-barriossistematizacion-de-experiencias/

Sabatini, F. (2006). La segregación social del espacio en las ciudades de América Latina. Washington, DC: Banco Interamericano de Desarrollo. https://publications.iadb.org/ en/publication/15146/la-segregacion-social-del-espacio-en-las-ciudades-de-americalatina 
Sabatini, F., Cáceres, G., \& Cerda, J. (2001). Segregación residencial en las principales ciudades chilenas: Tendencias de las tres últimas décadas y posibles cursos de acción. Revista EURE, 27(82), 21-42. http://doi.org/10.4067/S0250-71612001008200002

Salcedo, R., Sabatini, F., \& Rasse, A. (2009). Ciminalidad, control social e individualismo: reflexiones en torno a los cambios culturales en el habitar popular. En A. Lunecke, A. M. Munizaga, \& J. C. Ruiz (eds.), Violencia y delincuencia en barrios: sistematización de experiencias (pp. 67-81). Santiago de Chile: Fundación Paz Ciudadana/Universidad Alberto Hurtado. https://pazciudadana.cl/biblioteca/documentos/violencia-y-delin cuencia-en-barrios-sistematizacion-de-experiencias/

Sampson, R. J. (2004). Neighborhood and community: Collective efficacy and community safety. New Economy, 11, 106-113. https://doi.org/10.1111/j.1468-0041.2004.00346.x

Sampson, R. J., Morenoff, J. D., \& Earls, F. (2000). Beyond social capital: Spatial dynamics of collective efficacy for children. American Sociological Review, 64, 633-660. https://doi. org/10.2307/2657367

Sampson, R. J., Morenoff, J. D., Jeffrey, D., \& Gannon-Rowley, T. (2002). Assessing "neighborhood effects": Social processes and new directions in research. Annual Review of Sociology, 28, 443-478. https://doi.org/10.1146/annurev.soc.28.110601.141114

Segovia, O. (2005). Habitar en conjuntos de vivienda social: ¡cómo construir identidad, confianza y participación social? En A. Rodríguez \& A. Sugranyes (eds.), Los con techo. Un desafio para la politica de vivienda social (pp. 79-97). Santiago de Chile: Ediciones SUR. http://www.sitiosur.cl/r.php?id=81

Skewes, J. C. (2005). De invasor a deudor: el éxodo desde los campamentos a las viviendas sociales en Chile. En A. Rodríguez \& A. Sugranyes (eds.), Los con techo. Un desafío para la politica de vivienda social (pp. 101-122). Santiago de Chile: Ediciones SUR. http:// www.sitiosur.cl/r.php?id=81

Small, M. L. (2004). Villa Victoria: The transformation of social capital in a Boston barrio. Chicago and London: University of Chicago Press.

Sugranyes, A. (2005). La política habitacional en Chile, 1980-2000: un éxito liberal para dar techo a los pobres. En A. Rodríguez \& A. Sugranyes (eds.), Los con techo. Un desafio para la politica de vivienda social (pp. 23-57). Santiago: Ediciones SUR. http://www. sitiosur.cl/r.php?id=81

Svendsen, G. \& Haase, L. (2010). Socio-spatial planning in the creation of bridging social capital: the importance of multifunctional centers for intergroup networks and integration. International Journal of Social Inquiry, 3(2), 45-73. http://dergipark.gov. tr/ijsi/issue/17731/185716

Tironi, M. (2003). Nueva pobreza urbana. Vivienda y capital social en Santiago de Chile 19852001. Santiago de Chile: Predes/Ril Editores.

Tironi, M. (2009). The lost community? public housing and social capital in Santiago de Chile, 1985-2001. International Journal of Urban and Regional Research, 33(4), 974-997. https://doi.org/10.1111/j.1468-2427.2009.00893.x

Vidal, T. \& Pol, E. (2005). La apropiación del espacio: una propuesta teórica para comprender la vinculación entre las personas y los lugares. Anuario de Psicología, 36(3), 281-297. https://www.raco.cat/index.php/anuariopsicologia/article/viewFile/61819/81003 